\title{
Retrospective Testing of the Score for the Detection of Ectasia Susceptibility: A Case Report of Ectasia 7 Years after LASIK
}

\section{Alain Saad, Damien Gatinel}

\begin{abstract}
A patient was treated bilaterally for myopia using laser in situ keratomileusis (LASIK). There was no evident tomographic risk factor for ectasia, but a slight asymmetry on the Placido topography in the left eye. Seven years postoperatively, a unilateral ectasia was diagnosed. Retrospectively, the calculated Score for the left eye was +0.2 . This case suggests that an artificial intelligence system based on the combination of Placido and tomographic indices may be helpful for the detection of ectasia susceptible eyes.
\end{abstract}

Keywords: Lasik, Ectasia, Keratoconus detection, Keratoconus suspect, Score.

How to cite this article: Saad A, Gatinel D. Retrospective Testing of the Score for the Detection of Ectasia Susceptibility: A Case Report of Ectasia 7 Years after LASIK. Int J Kerat Ect Cor Dis 2013;2(2):73-78.

\section{Source of support: Nil}

Conflict of interest: None declared

\section{INTRODUCTION}

Keratectasia is an uncommon but severe complication of laser in situ keratomileusis (LASIK). As the number of patients who have refractive surgery increases, more cases of ectasia are being recognized..$^{1-5}$ The best predictor for the development of ectasia after LASIK is the presence of an undetected ectatic disorder preoperatively, for which LASIK is an aggravating factor through the acceleration of the ectatic process. ${ }^{2}$ Ectasia usually occurs within the first year following surgery, although cases have been reported to occur up to 9 years postoperatively. ${ }^{5}$ We recently described a new method named Score for the detection of very early form of keratoconus (ectasia susceptible corneas). ${ }^{6-8}$ In this artificial intelligence system based on the linear discriminant analysis of multiple quantitative topographic indices, a positive Score $(>0)$ suggest a suspicious cornea while a negative one $(<0)$ suggest a normal cornea. We present the case of a patient treated for myopia who developed unilateral corneal ectasia 7 years after LASIK. The retrospectively calculated Score was slightly positive for the eye that developed an ectatic process.

\section{CASE REPORT}

A 31-year-old woman presented for refractive surgery at our institution in July 2005. The corrected distance visual acuity (CDVA) was 20/20 in both eyes with a refraction of $-4.50(-0.25 \times 110)$ Diopters (D) and $-4.50(-1.00 \times 80)$ $\mathrm{D}$ in the right eye and left eye, respectively. At the time of consultation, the refraction had been stable for more than 2 years. The patient had no family history of keratoconus and no personal history of allergy or eye rubbing. Biomicroscopy and fundoscopy were normal. Corneal topography obtained with the Orbscan II (Technolas Perfect Vision, Munich, Germany) is shown in Figures 1A and B. The mean central pachymetry was $541 \mu \mathrm{m}$ in the right eye (thinnest point $526 \mu \mathrm{m}$ ) and $518 \mu \mathrm{m}$ in the left eye (thinnest point $512 \mu \mathrm{m}$ ).

The patient had bilateral LASIK surgery in October 2005, without intraoperative complications. A microkeratome (Hansatome, Bausch \& Lomb) was used to create the flap. The intended flap thickness was $160 \mu \mathrm{m}$ and the flap diameter was $8.5 \mathrm{~mm}$. Laser ablation was performed using a Nidek EC5000 (Nidek, Japan). One month postoperatively, the uncorrected distance visual acuity (UDVA) was 20/20 in the right eye and 20/25 in the left eye. Best corrected distance visual acuity (BCDVA) was 20/20 in the left eye with a correction of $-0.50(-0.75 \times 170)$. Four months postoperatively, in February 2006, the VA was unchanged in the right eye and was 20/20 in the left eye with a correction of $+0.25(-1.00 \times 155)$. An enhancement was performed in April 2006 and plano $(-1.00 \times 155)$ was programmed in the laser. She consulted for the last time in April 2006 after the enhancement and her visual acuity was 20/20 (plano) in the right and left eyes.

In June 2013, 7 years after her surgery, the patient presented with blurring of vision in the left eye. The symptoms had started about few months ago. The UCDVA was $20 / 20$ in the right eye and the BCDVA was 20/200 in the left eye. Combined Placido and elevation topography (Figs 2A and B) showed an important paracentral steepening suggestive of corneal ectasia in the left eye, while topography of the right eye was normal. Anterior segment optical coherence tomography (Visante OCT, Zeiss) showed a flap thickness of $125 \mu \mathrm{m}$ with a residual stromal bed of $386 \mu \mathrm{m}$ in the right eye and $127 \mu \mathrm{m}$ and $373 \mu \mathrm{m}$, respectively, in the left eye (Figs 3A and B).

In order to improve his quality of vision, we referred the patient for rigid contact lens adaptation. 

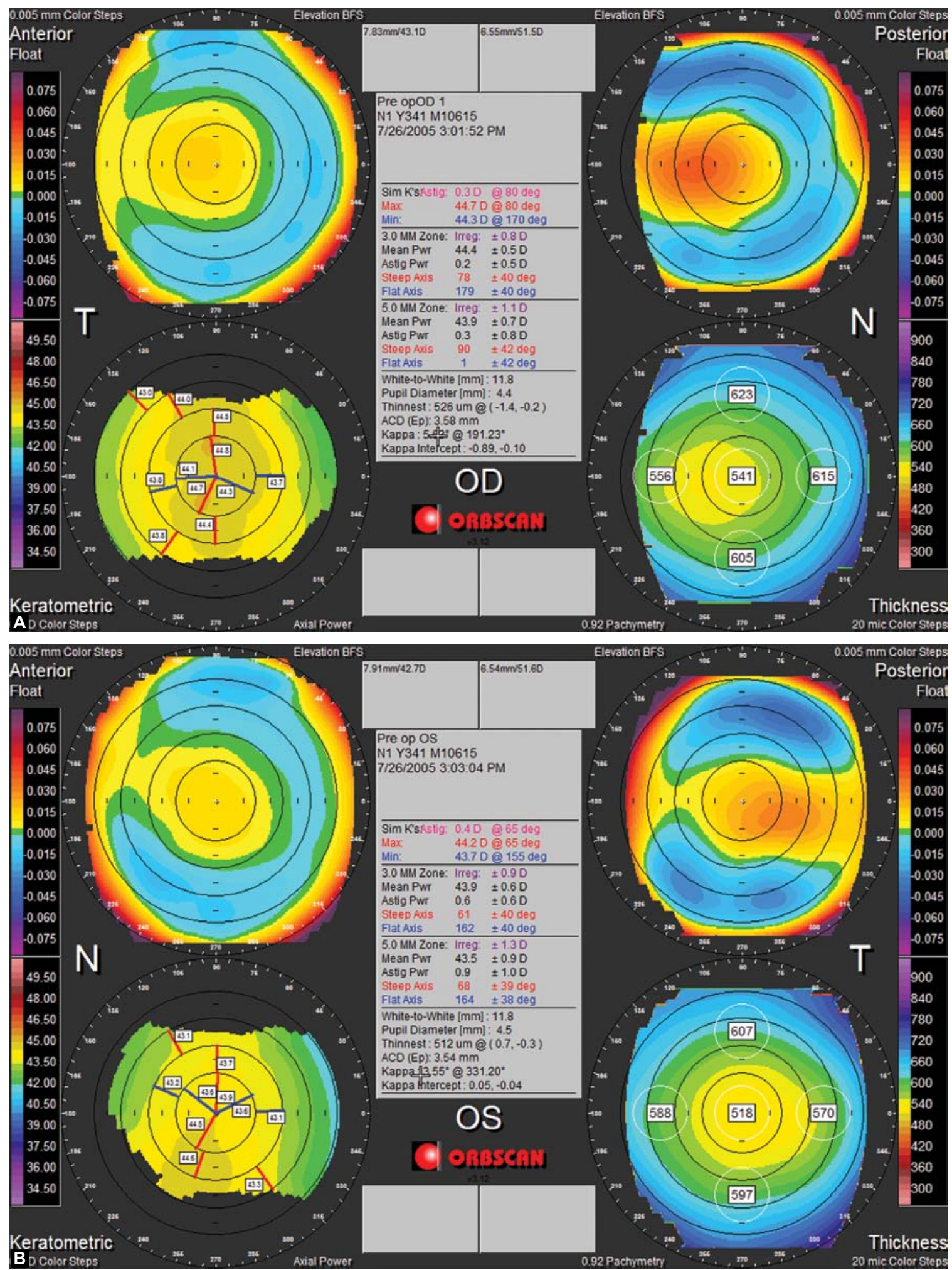

Figs $1 A$ and B: Preoperative Placido and slit scanning topography (QuadMap) of the right (A) and left (B) corneas

\section{DISCUSSION}

The preoperative Placido topographies of our patient revealed a regular cornea in the right eye and a slight superior - inferior (I-S) asymmetry in the left eye (Figs $1 \mathrm{~A}$ and B). However, the I-S was 0.9D in the left eye, which is below the suspicion threshold. In addition, anterior and posterior elevation looks normal in both eyes and there was no decentration of the thinnest point. The cornea was below the detection threshold of the Corneal Navigator System of the OPD scan (Figs 4A and B) which relies on multiple corneal indices obtained from the Placido topography. At the time of the surgery, the subjective evaluation of the cornea did not exclude this patient from having corneal refractive surgery. 

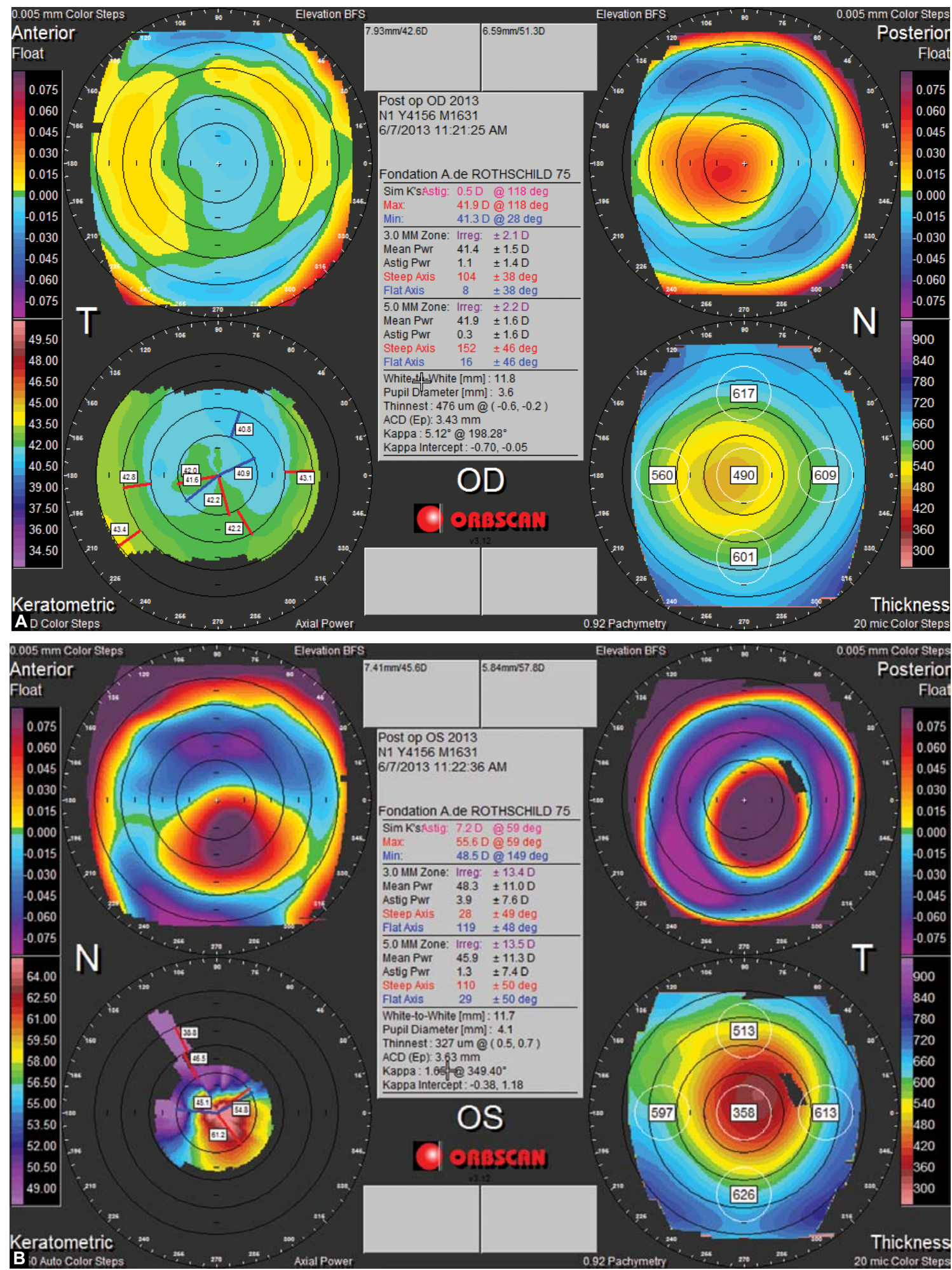

Figs 2A and B: Placido and slit scanning topography (QuadMap) of the right (A) and left (B) corneas 7 years postoperatively

Ectasia remains a mysterious complication of refractive surgery and the reason why it happens in some cases is sometime unexplained. An undetected preoperative subclinical keratoconus seems to be the most important predictor factor however, there is not a clear consensus on the definition of subclinical keratoconus. ${ }^{2,7,9}$ Some people defined subjectively the subclinical keratoconus based on topographic and tomographic evaluation of the cornea. ${ }^{10}$ Other relies on objective Placido-based parameters such as the I-S, the SRAX or the KISA for a better classification. ${ }^{11-15}$ All the used denominations (subclinical keratoconus, forme fruste keratoconus, keratoconus suspect) can be confusing, and have been employed interchangeably; however, in clinical practice, the main goal of the refractive surgeon 

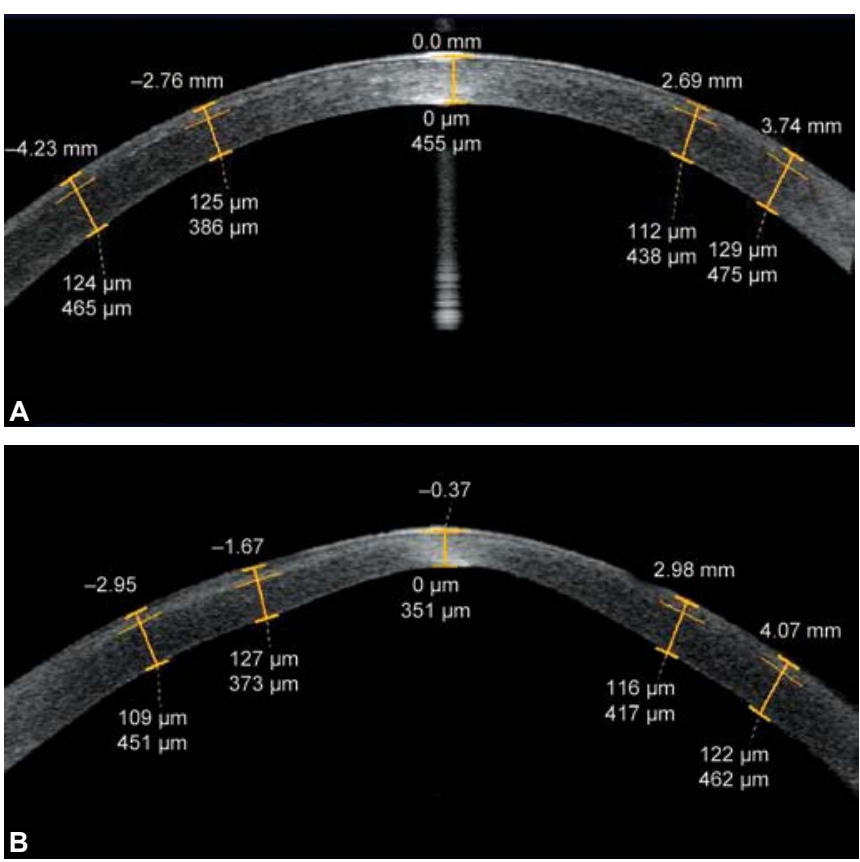

Figs $3 A$ and $B$ : Anterior segment optical coherence tomography of the right $(A)$ and left $(B)$ corneas 7 years postoperatively

remain to evaluate the ectasia susceptibility of refractive surgery candidates.

Most iatrogenic ectasia appears much earlier, and to our knowledge, this is one of the latest reported cases of postLASIK ectasia occurring more than 6 years after surgeries. ${ }^{1,5}$ Pregnancy was described as a potential trigger factor for iatrogenic ectasia ${ }^{5}$ and it is noteworthy that our patient delivered 12 months before the appearance of the symptoms.

It is supposed that the massive increase in serum estrogen levels in late pregnancy with the presence of estrogen receptors in the human cornea may be responsible for increasing the risk of iatrogenic ectasia in predisposed individuals. ${ }^{5}$ Spoerl et $\mathrm{a}^{16}$ have demonstrated that ex vivo porcine corneas show a distinct reduction in biomechanical stiffness when exposed to high doses of estradiol. However, in our patient, the symptoms did not appear during the pregnancy but few months later. Hafezi et $\mathrm{al}^{5}$ described 5 patients with iatrogenic ectasia after pregnancy. In all their cases, the symptoms were concomitant to the pregnancy which makes it easier to conclude to a causal effect of the pregnancy.

Perhaps the importance of the preoperative abnormality / suspicion plays a role in the delay between the surgery (and the pregnancy) and the appearance of the ectasia, meaning that highly abnormal corneas may develop much faster post -LASIK ectasia.

We previously described a new method for the automated detection of ectasia susceptible eyes-based on the evaluation of the contralateral topographically normal eye of 'unilateral' keratoconus. ${ }^{6-8}$ This method is based on
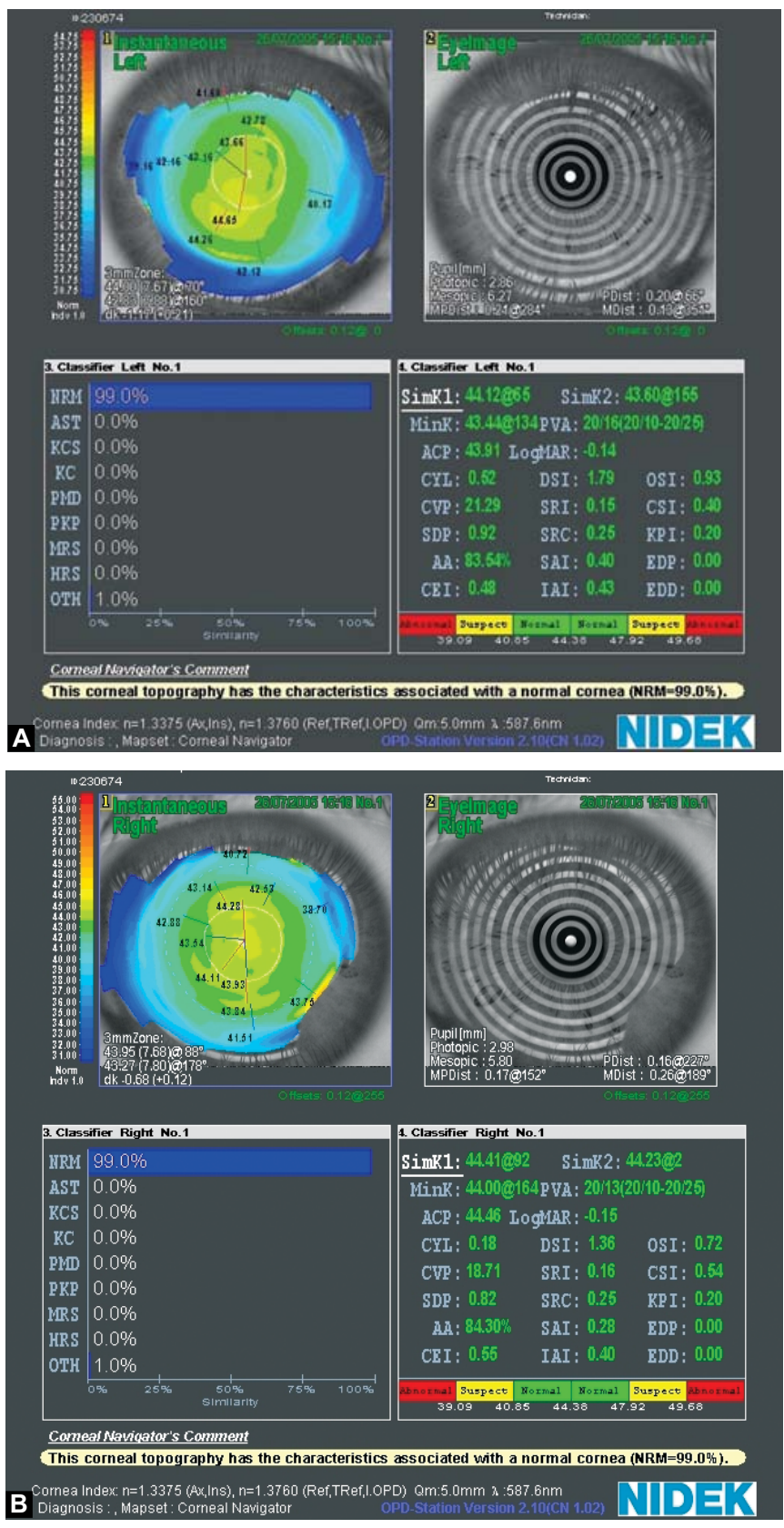

Figs 4A and B: Preoperative Placido topography and corneal navigator system classification of the right $(A)$ and left $(B)$ corneas

discriminant analysis, and incurs the linear combination of 12 quantitative indices obtained from Orbscan's raw data. It has been incorporated in an automated software named Score. To our knowledge, this scoring system is the only artificial intelligence that combines Placido and tomographic indices in a weighted fashion to classify the corneas as suspect or normal.

The Score of the left eye was +0.2 while the score of the right eye was -2.2 (Figs $5 \mathrm{~A}$ and $\mathrm{B}$ ). A negative score reflects a normal cornea while a positive score reflects a suspicious one. Thus, when the score is positive, LASIK should be contraindicated, while PRK can still be considered, based on individual case and some other parameters evaluation. 


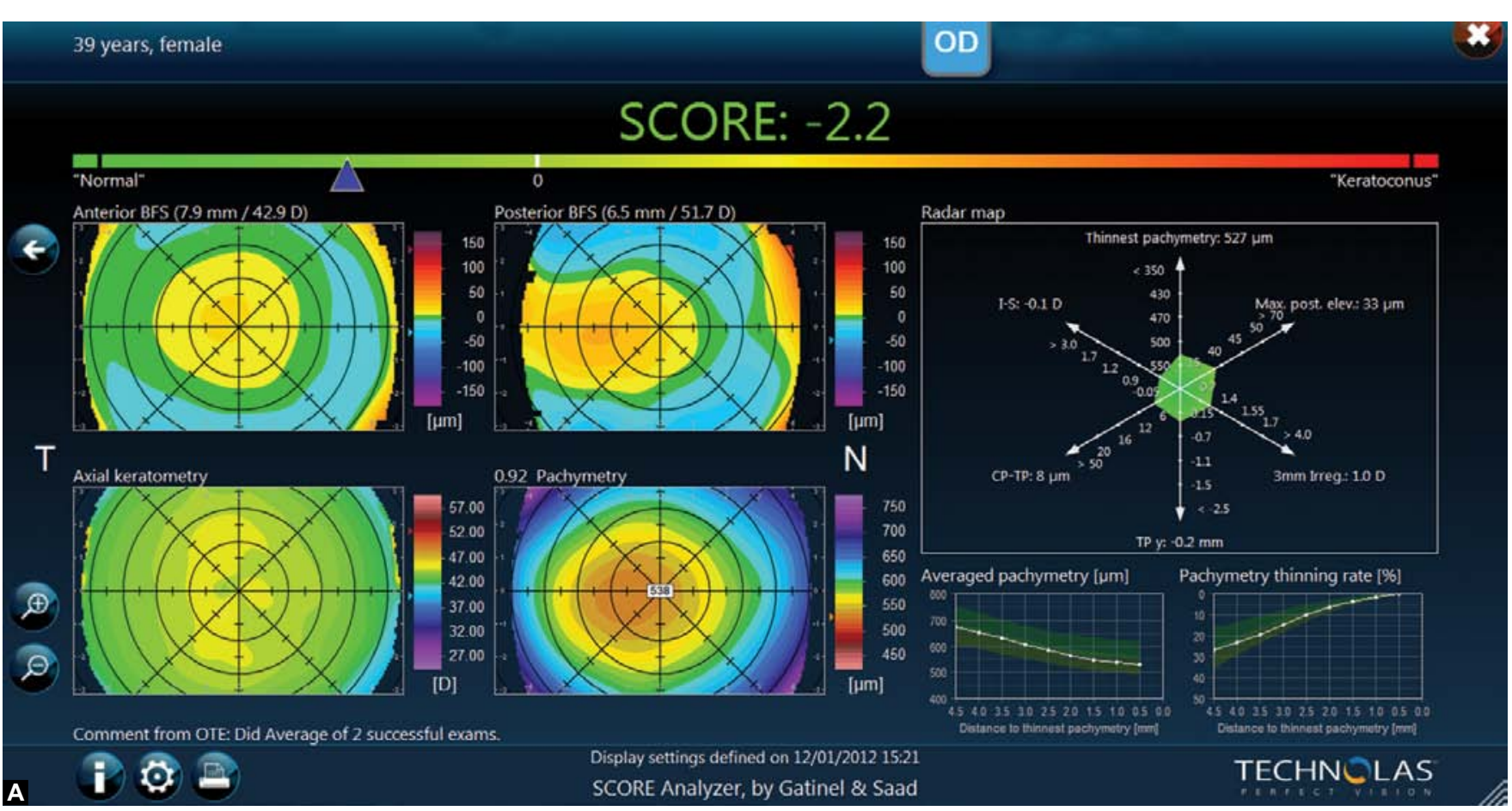

39 years, female

SCORE: 0.2

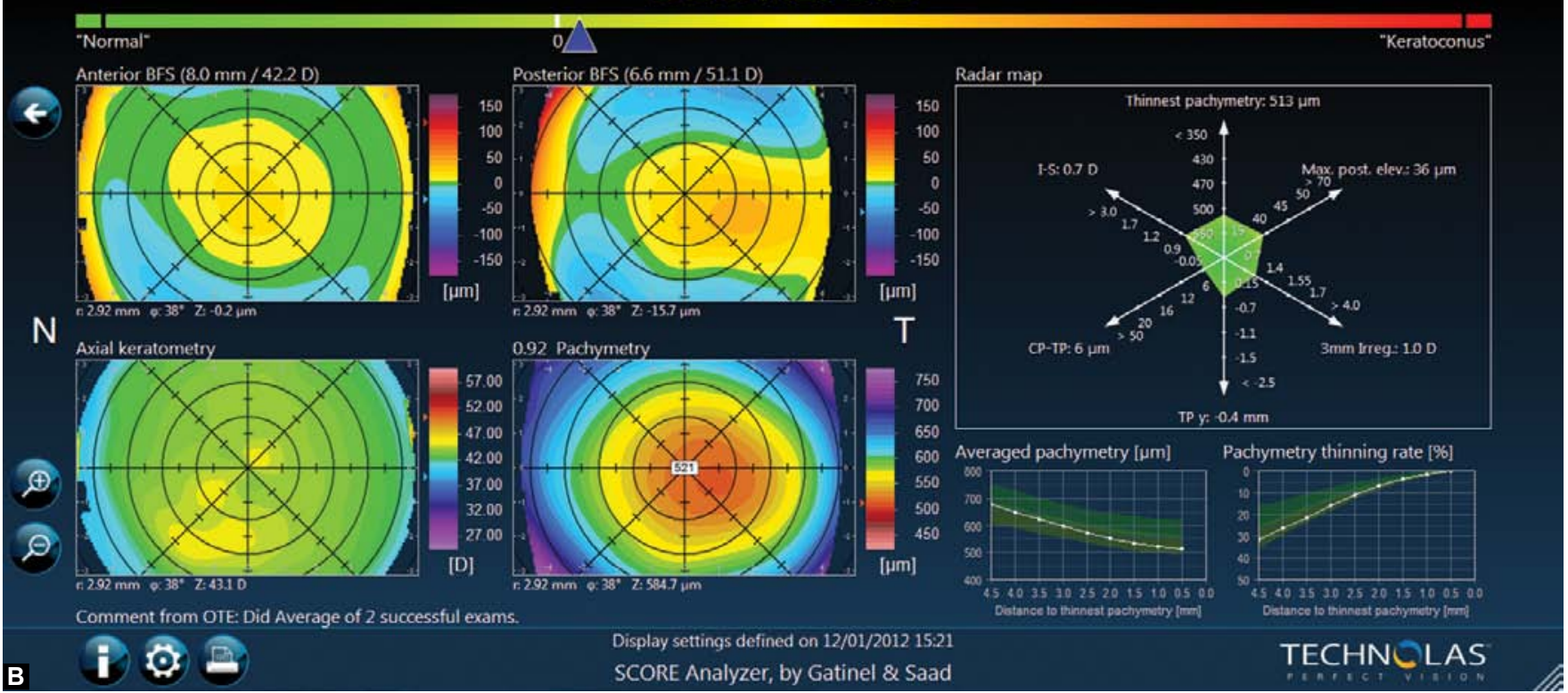

Figs $5 A$ and $B$ : Score of the right $(A)$ and left $(B)$ corneas

Figures 5A and B shows the display of the Score. In addition to the score value, two graphs and a radar map describe the most discriminant indices. In the left eye, all the indices were borderline (at the limit between green color and yellow one) which lead to a slightly positive final score. The pachymetry thinning rate was also limit but still within the two standard deviation of a normal population. The importance of combining all the information is highlighted here: despite the fact that each specific index may be normal when considered separately, the combination of several indices in a discriminant function can result in diagnosing a suspicion cornea. The I-S index and the pachymetry thinning rate were different between right and left eye of this patient, which certainly contributes to the difference in the final score between the two eyes.

Analyzing preoperative data of corneas having iatrogenic ectasia represent the best method to validate the sensitivity of an artificial intelligence system aimed to evaluate ectasia susceptibility. ${ }^{17}$ Evaluation on larger group remains necessary to drive clear conclusions. However, such groups are difficult to form as the incidence of ectasia remains very low. 
In the future, this approach could be further extended to include biomechanical indices and other topographic data, such as epithelial thickness mapping to improve our ability to prevent iatrogenic ectasia.

\section{REFERENCES}

1. Spadea L, Cantera E, Cortes M, Conocchia NE, Stewart CW. Corneal ectasia after myopic laser in situ keratomileusis: a longterm study. Clin Ophthalmol 2012;6:1801-1813.

2. Randleman JB, Woodward M, Lynn MJ, Stulting RD. Risk assessment for ectasia after corneal refractive surgery. Ophthalmology 2008; 115:37-50.

3. Guilbert E, Saad A, Gatinel D. Unilateral ectasia after LASIK in a patient with abnormal topography but normal tomography. J Refract Surg 2013;29:294-296.

4. Saad A, Gatinel D. Bilateral corneal ectasia after laser in situ keratomileusis in patient with isolated difference in central corneal thickness between eyes. J Cataract Refract Surg 2010;36:1033-1035.

5. Hafezi F, Koller T, Derhartunian V, Seiler T. Pregnancy may trigger late onset of keratectasia after LASIK. J Refract Surg 2012;28:242-243.

6. Saad A, Gatinel D. Topographic and tomographic properties of forme fruste keratoconus corneas. Invest Ophthalmol Vis Sci 2010;51:5546-5555.

7. Gatinel D, Saad A. The challenges of the detection of subclinical kerateconus at its earliest stage. Int J Keratoconus and Ectatic Corneal Diseases 2012;1:36-43.

8. Saad A, Gatinel D. Validation of a new scoring system for the detection of early forme of keratoconus. Int $\mathrm{J}$ Keratoconus and Ectatic Cor Dis 2012;1:100-108.

9. Saad A, Gatinel D. Subclinical keratoconus, keratoconus suspect, forme frustre keratoconus - the need for an objective classification system. Ophthalmol 2013; In Press.

10. Arbelaez MC, Versaci F, Vestri G, Barboni P, Savini G. Use of a support vector machine for keratoconus and subclinical keratoconus detection by topographic and tomographic data. Ophthalmol 2012;119:2231-2238.

11. Klyce SD. Chasing the suspect: keratoconus. Br J Ophthalmol 2009;93:845-847.

12. Klyce SD, Karon MD, Smolek MK. Screening patients with the corneal navigator. J Refract Surg 2005;21:S617-622.

13. Klyce SD, Smolek MK, Maeda N. Keratoconus detection with the KISA\% method-another view. J Cataract Refract Surg 2000;26:472-474.

14. Li X, Yang H, Rabinowitz YS. Keratoconus: classification scheme based on videokeratography and clinical signs. J Cataract Refract Surg 2009;35:1597-1603.

15. Rabinowitz YS, Rasheed K. KISA\% index: a quantitative videokeratography algorithm embodying minimal topographic criteria for diagnosing keratoconus. J Cataract Refract Surg 1999;25:1327-1335.

16. Spoerl E, Zubaty V, Terai N, Pillunat LE, Raiskup F. Influence of high-dose cortisol on the biomechanics of incubated porcine corneal strips. J Refract Surg 2009;25:S794-798.

17. Ambrosio R Jr, Randleman JB. Screening for ectasia risk: what are we screening for and how should we screen for it? J Refract Surg 2013;29:230-232.

\section{ABOUT THE AUTHORS}

\section{Alain Saad}

Ophthalmologist, Department of Cornea, Cataract and Refractive Surgery, Rothschild Foundation, Paris, France

Correspondence Address: Fondation Ophthalmologique Adolphe de Rothschild, 25, Rue Manin-75019, Paris, France, Phone: 33-148036486 Fax: 33-148036487, e-mail: dralainsaad@gmail.com

\section{Damien Gatinel}

Chief, Department of Cornea, Cataract and Refractive Surgery Rothschild Foundation, Paris, France 